\title{
Cancer Therapy with Plasmonic Nanoparticles
}

\author{
Ji-Ho Park* \\ Department of Bio and Brain Engineering, KAIST, Daejeon 305-701, Republic of Korea \\ (jihopark@kaist.ac.kr)
}

\begin{abstract}
A significant barrier to the clinical translation of systemically administered therapeutic nanoparticles is their tendency to be removed from circulation by the mononuclear phagocyte system. The addition of a targeting ligand that selectively interacts with cancer cells can improve the therapeutic efficacy of nanomaterials, although these systems have met with only limited success. Current approaches to nanoparticle targeting lack mechanisms of communication and amplification through which specific targeting events could assist the targeting of materials still in circulation. By contrast, natural systems frequently improve the efficiency of targeting through communication and cooperation between multiple components in a system. In this talk, inspired by such biological systems, we built a 'nanosystem' comprised of two distinct nanoparticle populations (plasmonic nanoparticles and drug nanocarriers) that communicate and cooperate each other in vivo to amplify drug or imaging payload delivery. We construct a cooperative nanosystem consisting of two discrete nanomaterials. The first component is plasmonic gold nanorod "activators" that populate the porous tumor vessels and act as photothermal nanoantennas to specify tumor heating via remote near-infrared laser irradiation. We find that local tumor heating accelerates the recruitment of the second component: a targeted nanoparticle consisting of either magnetic nanoworms or doxorubicin loaded liposomes. This study demonstrates that the appropriate combination of nanomaterials currently under investigation in cancer therapy can significantly enhance therapeutic efficacy relative to the individual components. Site-specific photothermal heating of plasmonic gold nanorods can engineer the local tumor microenvironment to enhance the accumulation of therapeutic targeted liposomes, which increases the overall hyperthermal and chemotherapeutic tumor-destroying effects. Cooperative, synergistic therapies using plasmonic properties of the nanoparticles could significantly reduce the required dose of anticancer drugs, mitigating toxic side effects, and more effectively eradiating drugresistant cancers.
\end{abstract}

